This item was submitted to Loughborough's Research Repository by the author.

Items in Figshare are protected by copyright, with all rights reserved, unless otherwise indicated.

\title{
Pathways within dementia diagnosis
}

\section{PLEASE CITE THE PUBLISHED VERSION}

https://he.palgrave.com/page/detail/Living-With-Dementia/?K=9781137593740

\section{PUBLISHER}

Red Globe Press () The Authors

VERSION

AM (Accepted Manuscript)

\section{PUBLISHER STATEMENT}

This book chapter was accepted for publication in the book Living With Dementia: Relations, Responses and Agency in Everyday Life and the definitive published version is available at https://he.palgrave.com/page/detail/Living-With-Dementia/?K=9781137593740

\section{LICENCE}

CC BY-NC-ND 4.0

\section{REPOSITORY RECORD}

Plejert, Charlotta, Danielle Jones, and Elizabeth Peel. 2017. "Pathways Within Dementia Diagnosis". figshare. https://hdl.handle.net/2134/27459. 
Plejert, C., Jones, D. \& Peel, E. (2017). Pathways within dementia diagnosis: Conversation Analytical perspectives. In L-C. Hydén \& E. Antelius (Eds.) Life with Dementia: Relations, Responses and Agency in Everyday Life. London: Palgrave Macmillan. (C)

\section{Pathways within dementia diagnosis}

Charlotta Plejert, Danielle Jones and Elizabeth Peel

\section{Introduction}

In this chapter, we foreground different dementia diagnostic pathways across three temporal phases of the process of diagnosis; from initial history taking in memory clinic assessment, to the disclosure and discussion of diagnosis. Therefore we are interested in, not only pathways to a diagnosis of dementia but rather the pathways and experiences within the diagnostic process, exploring the interactions that occur during this process. Specifically we focus on: first, conversational profiling and differential diagnosis during memory clinic assessment; second, interpreter-mediated dementia evaluations; and third disclosure and discussion of diagnosis.

A dementia diagnosis is a life-changing and often overwhelming experience, both for the person who receives the diagnosis, and for relatives and other people close. For some, however, getting a diagnosis may also be a relief, because it offers an explanation for worrying symptoms. Pathways towards a diagnosis, however, vary a great deal from person to person and family to family. In western societies at least, with growing cultural awareness of dementias, public health campaigns, and heightened media profile (Peel, 2014) increasing numbers of people and their families are seeking a formal dementia diagnosis. In the early stages, signs such as mild forgetfulness and other cognitive and behavioural changes may be thought of as indicators of stress or depression, or caused by factors other than a dementia disease. It might also be the case that the person with dementia is not able to self-monitor and therefore perceives symptoms of the disease differently from their family and friends. This may also affect help-seeking behaviours. In addition, various cultural belief-systems affect if, when, and to what extent people turn to health-care services. Whereas most people in cultures influenced by a biomedical perspective view dementia as an illness, there are also groups and populations that treat dementia symptoms as a result of many things, ranging from natural signs of ageing, madness, or even enlightenment (e.g. Dilworth-Anderson \& Gibson, 2002; Leibing, \& Cohen, 2006). Some studies have observed differences between populations (in the US) concerning the duration from the point of first notice and symptom recognition to final diagnosis, suggesting that people of a certain ethnicity, for example Afro-American and Hispanic people, have a longer lag time between first notice and diagnosis than white Americans (e.g. Schrauf \& Iris, 2011). An explanation to this difference might be reflected in how families choose to deal with symptoms. In the study by Schrauf and Iris (2011), it was claimed that Hispanic and African American caregivers "effectively provided a kind of 'scaffolding' for the patient" (p.743), which was a way of actively dealing with the disease, but also affected the length of time before seeking medical attention. This potentially also explains why, for example, African Americans show greater symptom severity at the time of diagnosis than some other populations (Dilworth-Anderson et al., 2002, Shadlen et al., 1999). 
Plejert, C., Jones, D. \& Peel, E. (2017). Pathways within dementia diagnosis: Conversation Analytical perspectives. In L-C. Hydén \& E. Antelius (Eds.) Life with Dementia: Relations, Responses and Agency in Everyday Life. London: Palgrave Macmillan. (C)

In addition to issues related to ethnicity, several other socio-cultural influences inform people's understanding and experience of diagnosis, for example gender (Erol, et al., 2015, 2016), sexual identity (Peel \& McDaid, 2015), age (Rosser et al., 2010), and social class (O’Connor et al., 1991). There is also geographical variability in dementia service provision, both nationally and internationally, which impacts both access to and the experience of services and adequate care. Differences in diagnostic procedures may also vary, for example whether diagnostic testing occurs in primary or secondary care or whether pre-diagnostic counselling and post-diagnostic support is offered. These modes of service delivery and structural health provision issues make a difference in terms of referral rates to access further testing, the time people have to wait to access memory services, and likely their experience of the process.

Once a person who is suspected to have a dementia disease seeks care, s/he will undergo dementia assessment. The diagnostic process comprises numerous different parts, ordinarily consisting of a combination of history-taking (medical interview), formal tests of cognitive functioning, physical and psychiatric examinations, and ancillary investigations, blood tests, brain scans and lumbar punctures. Sometimes further investigations may be needed, such as extended neuropsychological testing, particularly if screening tests are normal or equivocal, and there is still a concern about cognitive and behavioural changes (Adelman \& Daly, 2005).

The processes involved in a dementia evaluation are of great importance in several ways. It is not just a matter of reaching an accurate diagnosis, but the actual practices that occur between clinicians, patients and patients' companions, are key for how pathways within dementia diagnosis is experienced. Patient satisfaction with the diagnostic process must be achieved. Building an enabling and trusting relationship with the clinicians in charge of the evaluations is an important part of turning an already challenging situation, into something less emotionally taxing, especially as patients and carers view the diagnostic process as labyrinthine (Samsi et al., 2013) and (similarly) subsequent access to services as akin to navigating a maze (Peel \& Harding, 2014).

Research on dementia diagnostic practices has so far primarily been carried out within the field of medicine. More recently, however, it has become a focus in social scientific research, particularly that which examines what happens in patient-doctor encounters. To date, this line of research has explored, for example, how people talk about their symptoms, and how the occurrence of specific interactional features can be used to assist in the diagnosis of memory complaints (Elsey et al., 2015; Jones et al., 2016), and how a diagnosis is delivered and received (c.f. Heritage \& Maynard, 2006; Lecouturier et al., 2008; Maynard \& Frankel, 2006). Recent research has also highlighted specific aspects of dementia evaluations for ethnic minority patients, where an interpreter is needed in order to mediate talk between clinical staff and patients and their companions (Plejert et al., 2015; Majlesi \& Plejert, 2016). It is through a social scientific lens that we approach the pathways within dementia diagnosis that are the focus of this chapter. 
Plejert, C., Jones, D. \& Peel, E. (2017). Pathways within dementia diagnosis: Conversation

Analytical perspectives. In L-C. Hydén \& E. Antelius (Eds.) Life with Dementia: Relations, Responses and Agency in Everyday Life. London: Palgrave Macmillan. (C)

\section{Conversation Analysis}

One way of understanding the processes involved in dementia diagnosis, is to study the practices and actions that are established in, and through, interaction between the participants at hand. For this purpose, the framework of Conversation Analysis (CA; e.g. Sidnell \& Stivers, 2013; Schegloff, 2007) has proven useful, not only for the study of dementia (e.g. Chatwin, 2014), but also other conditions that affect language and interaction (e.g. Goodwin, 2003).

CA has its origin in sociology and ethnomethodology and is a theoretically and methodologically distinctive approach to the study of talk-in-interaction. The principle objective of CA is to discover the patterns, mechanisms and practices of interaction, which underlie our social competences, by examining recorded instances of naturally occurring interactions. These recordings prove suitable for repeated examination and have revealed the cumulative empirical findings of the discipline, for example turn taking (Sacks et al., 1974), turn design (Drew, 2015), sequence organisation (Schegloff, 2007), repair (Schegloff et al., 1977) and social action (Schegloff, 1996). Today, video recordings serve as the basis for most analyses, since they provide access to both verbal and non-verbal features of interactions, which can be vital when investigating and understanding social conduct. CA has been applied successfully in a variety of primary and secondary medical services, exploring the features and practices within a range of medical communications. These studies have informed medical practice and have altered certain diagnostic processes (Robson et al., 2012; Heritage et al., 2007; Stivers, 2007). This way of feeding back into the areas under investigation is particularly typical for the branch commonly referred to as "Applied CA" (see Antaki, 2011). The three areas presented in the sections that follow, may all be viewed as representative of this applied approach to $\mathrm{CA}$.

\section{Conversational profiling and differential diagnosis during memory clinic assessments}

In many countries world-wide, there is an increase in the number of people referred to memory clinics and other specialist services. This is partially due to a raised awareness of the benefits of an early or "timely" diagnosis (Brooker et al., 2014) for accessing health and social care interventions, enabling future care planning and increasing the efficacy of both drug and non-drug treatments. This development is also true for the UK, where referral rates are already high, and expected to rise even further (Royal College of Psychiatrists, 2013). A problem that arises from this significant increase in patients referred to memory clinics is the pressure to provide both a speedy and an accurate diagnosis; this presents challenges for specialist practitioners. One specific difficulty is the need to distinguish dementia from other forms of psychological, cognitive, or further memory problems that display similar symptomology, e.g. depression, trauma, mild cognitive impairment (MCI), and functional memory disorder (FMD). The differentiation between diagnoses relies on a lengthy evaluation process, meaning that it can be a difficult and time-consuming procedure. It may 
Plejert, C., Jones, D. \& Peel, E. (2017). Pathways within dementia diagnosis: Conversation Analytical perspectives. In L-C. Hydén \& E. Antelius (Eds.) Life with Dementia: Relations, Responses and Agency in Everyday Life. London: Palgrave Macmillan. (C)

also be the case that test results are equivocal. Additional tools that could be used to assist in the diagnostic process are therefore needed, particularly if they could speed up the diagnostic process, and decrease the need for complex further neuropsychological testing that can result in anxiety for patients. One relevant aspect of these concerns deals with how experienced clinicians often create a "working diagnosis", formed on the basis of their initial impressions of the first few minutes of conversation with a patient with memory problems (Jones et al., 2016). The exact nature of this working diagnosis is of great interest for CA-researchers, who have the tools for providing insights on how this diagnosis comes about, and to what extent it is valid. Previous research on differentiating between epileptic and non-epileptic seizures suggests that analyses of how patients describe their problems (what they say or the content of their talk), for example using certain metaphors, as well as how they answer questions, may contain important information for their eventual diagnosis (e.g. Reuber et al., 2009; Plug et al., 2011) and may thus comprise elements that assist clinicians in distinguishing between different diagnoses. A similar approach has been used by Jones et al. (2016), who utilized CA-methodology for differentiating between patients with neurodegenerative dementia and FMD.

The study by Jones et al. (2016) represents an initial analysis of data from a larger study using CA in the memory clinic to identify potential interactional and linguistic diagnostic pointers. Jones et al. (2016) present findings based on the analysis of a sub-set of 25 videoand audio recordings of initial consultations between neurologists and patients referred to a specialist memory clinic in a UK city. Data were collected between 2012 and 2014. The patients were referred to the memory clinic through a variety of pathways including from primary care general practice (GPs) or other secondary care services such as non-specialist neurology and psychiatry. A 'gold standard' clinical diagnosis was made by three consultant neurologists with a special interest in memory disorders on the basis of a patient's initial visit, including screening with the Addenbrooke's Cognitive Examination (ACE-R), and subsequent detailed neuropsychological test battery and magnetic resonance imaging (MRI) of the brain. Nine of the patients included received a diagnosis of a neurodegenerative memory disorder (average ACE-R score 56/100, range from 28-80) and 16 were diagnosed with FMD (average ACE-R score 93/100 range from 85-99) (for a definition of FMD see Blackburn et al., 2014). Sixteen of the patients were female and 9 were male. The patients' ages ranged from 47 to 77 years. The initial history-taking phase of the encounters, which formed the basis of the analysis, lasted between 7 minutes and 28 seconds and 32 minutes and 29 seconds. None of the patients who ultimately received a diagnosis of a dementia attended the clinic alone, whereas 11 of the 16 patients with FMD came unaccompanied. Recordings were transcribed in considerable detail, using CA transcription conventions (Jefferson, 1983, 2004) and were analysed in accordance with CA-methodology. Initial analyses revealed five interactional features that appeared relevant for the purpose of differentiating between dementia and FMD. These features were: 
Plejert, C., Jones, D. \& Peel, E. (2017). Pathways within dementia diagnosis: Conversation Analytical perspectives. In L-C. Hydén \& E. Antelius (Eds.) Life with Dementia: Relations, Responses and Agency in Everyday Life. London: Palgrave Macmillan. (C)

1. Patients' abilities to answer questions concerning personal information, for example their age or where they lived.

2. If patients were able to display functional working memory from within the interaction itself.

3. If patients were able to respond to compound questions.

4. The time taken to respond to questions.

5. The level of specific details offered by patients when they gave accounts of their memory failure experiences.

Analyses of the first feature, responses to questions about personal information, revealed that people with FMD were able to produce answers both quickly, unproblematically and accurately. In contrast, people with dementia displayed difficulties producing correct information, which could lead to repair-sequences where the clinician, or companion had to repeat or rephrase the questions, or provide further explanatory information. For example, a question about age, was promptly answered with no delay by a person with FMD, whereas a person with dementia might initiate repair, either as a result of not comprehending the question, or confusing the question with something else, for example date of birth (cf. Jones et al., 2016, p. 502-3).

Another interactional feature that aided the process of differentiating patients with FMD from people with dementia was the display of working memory in interaction. Examples from conversations during the history taking part of the consultation demonstrated that people with FMD typically were able to display working memory both in relation to what someone else had previously said in the consultation, for example in terms of remembering and answering prior questions. People with FMD were also able to recall and repeat information that they had previously mentioned themselves, often after a significant time had passed. These latter accounts were also often marked by phrases such as "like I said" or "as I say". An example of this feature is illustrated in Excerpt 1, below. Transcription conventions follow Jefferson (2004) and are found at the end of the chapter.

Excerpt 1 (DOC=Doctor, PAT=Patient, OTH=Companion)

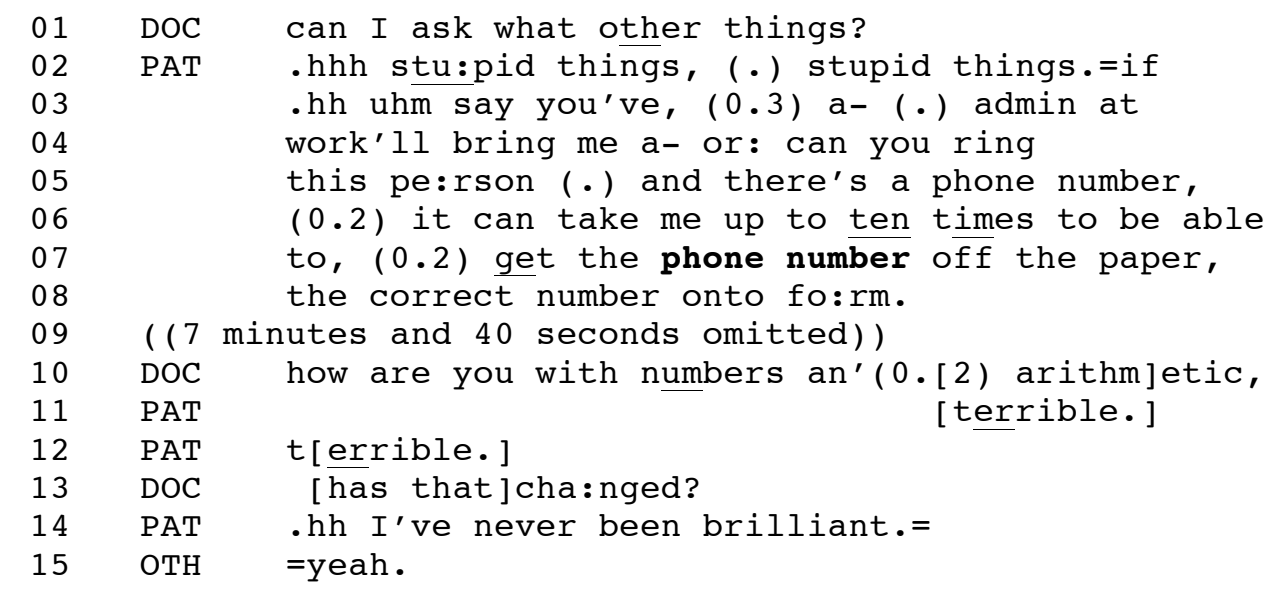


Plejert, C., Jones, D. \& Peel, E. (2017). Pathways within dementia diagnosis: Conversation Analytical perspectives. In L-C. Hydén \& E. Antelius (Eds.) Life with Dementia: Relations, Responses and Agency in Everyday Life. London: Palgrave Macmillan. (C)

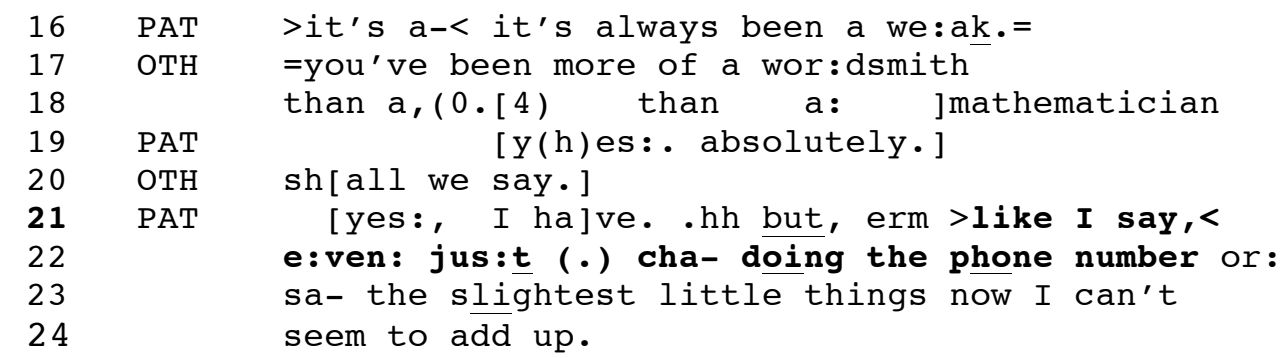

The doctor (line 01) is touching off a question based on the companion's previous assertion that "other things" about the patient's memory had been pointed out to them as being problematic. The patient produces an example about her inability to copy phone numbers down accurately as evidence of other memory problems she has experienced. Within the 7 minutes omitted from the transcript the patient finishes this discussion about her problem with phone numbers, with her husband likening her difficulty to 'dyslexia'. The consultation then goes on to discuss several unrelated issues such as the patient's work, when the problems started, the anxiety the patient experiences and issues to do with her spatial awareness. After this prolonged period of time the neurologist asks how the patient is with numbers (line 10). After admitting to be "terrible" (line 11-12), and the companion acknowledging the patient's proficiency with words rather than numbers (line 17-18), the patient again proceeds to use the example of her difficulty with phone numbers as evidence of how 'terrible' she is. Here the patient prefaces the repetition with "like I say" (line 21), to mark her self-repetition and to display that she is aware that the information she is producing has already been given earlier. This interactional resource, which displays working memory, appears as a feature in the interactions with patients with FMD and contributes to their conversational profile. Unlike the patients with FMD, people with dementia were often unable to display memories during consultations in the way exemplified in Excerpt 1. In addition, in cases where people with dementia repeated themselves, they did not display any markers that demonstrated that they were aware of their repetitions (e.g. "like I said"). Rather, repeated information was produced as if for the first time, something which has been demonstrated previously in CA-work on interaction and dementia (Hydén et al., 2012; Jones, 2013).

The third interactional feature documented by Jones et al., (2016) concerned patients' abilities to respond to compound or "multi-unit" questions (cf. Linell et al., 2003). This is a form of questioning that is very commonly used in institutional interaction, for example medical settings, court trials, police interrogations etc. In relation to potential memory problems, multi-unit questions are demanding, since they require that a person is able to remember several successive questions, and provide answers to each one of them. In the Jones' et al. study, people with FMD did not display any difficulties responding to multi-unit questions. In addition, they frequently also provided lengthy and detailed answers that covered what the clinician had asked about. People with dementia, however, demonstrated 
Plejert, C., Jones, D. \& Peel, E. (2017). Pathways within dementia diagnosis: Conversation Analytical perspectives. In L-C. Hydén \& E. Antelius (Eds.) Life with Dementia: Relations, Responses and Agency in Everyday Life. London: Palgrave Macmillan. (C)

difficulties comprehending questions, and had also problems recalling the different parts in their responses. Excerpt 2 provides an illustration of this feature.

Excerpt 2 (taken from Jones et al., 2016, p. 505)

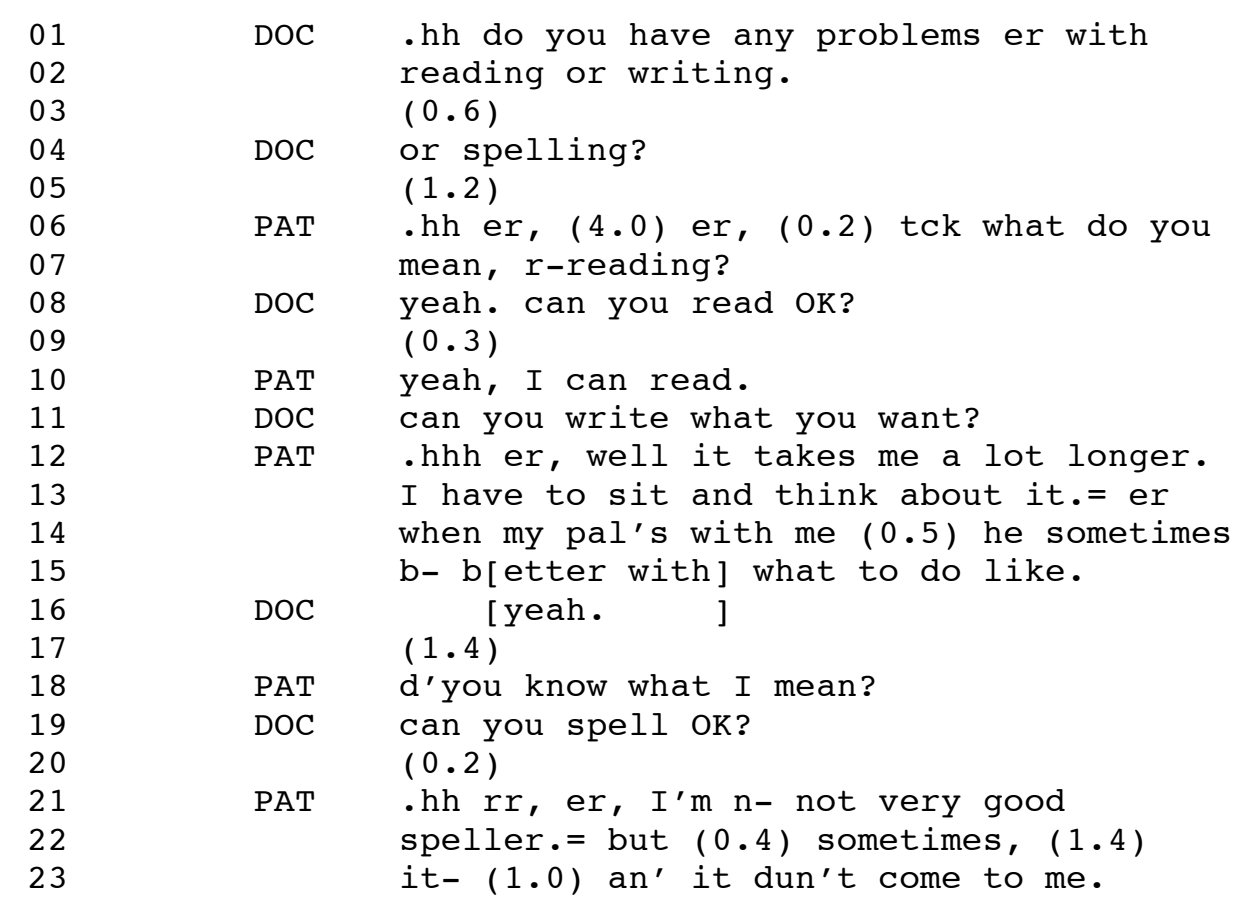

The patient does not accurately respond to all three parts of the multi-unit question (lines 0104) and instead, after a lengthy inter-turn gap (line 05) and turn initial pause (line 06), seeks clarification from the doctor (lines 06-07). The doctor re-issues the questions individually, after which the patient responds. This exemplifies the difficulties people with dementia displayed in recalling and processing multiple components simultaneously. The consultations with people with FMD did not reveal the same difficulty when responding to such multi-unit questions.

Delays in responding to questions were another interactional feature that distinguished people with FMD from people with dementia. The latter typically either did not respond to questions, which prompted further questioning or clarification from the clinician, or their responses were delayed, sometimes substantially (e.g. on occasion almost 10 seconds before the patient produced a response) and was therefore treated as noticeable and accountable by both the neurologist and accompanying person, who often sought to repair the situation.

The final feature dealt with patients' abilities to provide detailed accounts in their answers to the clinician's questions. When comparing the responses of the different patient groupings it was evident that people with FMD were often able to provide both lengthy and detailed answers, comprising new and unsolicited information, which displayed the person's episodic memory capabilities. Responses by people with dementia, however, often consisted of 
Plejert, C., Jones, D. \& Peel, E. (2017). Pathways within dementia diagnosis: Conversation Analytical perspectives. In L-C. Hydén \& E. Antelius (Eds.) Life with Dementia: Relations, Responses and Agency in Everyday Life. London: Palgrave Macmillan. (C)

admissions of forgetfulness or a lack of understanding, for example "Can't remember now" (Jones et al., 2016, p. 505). In contrast to the elaborated accounts provided by people with FMD, the answers by people with dementia were also very short single utterance-, or singleturn units that did not provide any additional or voluntary information. This is exemplified in Excerpt 3.

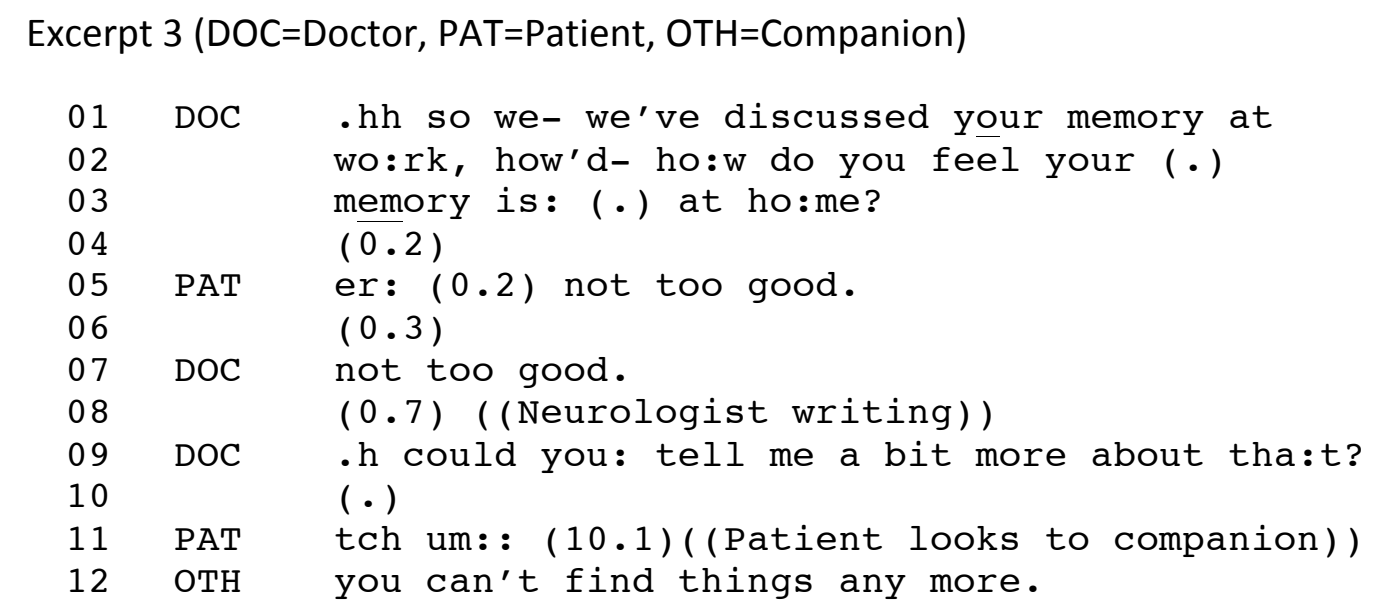

The neurologist is here closing the discussion about the patient's memory problems "at work" and focusing on additional issues he may face at home, seeking examples of the patients experiences (lines 01-02). After some delay the patient admits to his memory not being "too good" (line 05). The patient here does not elaborate on his response, providing any examples of instances where his memory failed. This unsolicited elaboration is often evident in cases where the person has FMD. Instead the neurologist pursues some elaboration by asking the patient to tell him "a bit more" about his memory failure experiences (line 09). The patient is unable to produce any detailed description of his memory problems and, after a lengthy delay of 10 seconds, turns to his companion for help. It is the companion who produces the detail required. These features, of people either not being able to answer a question (and thus relying on others to articulate the response on their behalf) or producing undetailed minimal responses, are recurrent interactional practices contributing to the interactional profiles of people with dementia.

The excerpts above have demonstrated different interactional patterns that can be used to distinguish dementia from FMD. In Jones et al. (2016), all participants were native English speakers. In the next section, we will present examples from a situation where the interaction between clinician and patient is mediated by an interpreter.

\section{Interpreter-mediated dementia evaluations}

As explicated above, the dementia evaluation is a complex procedure, where many different steps are taken, and different instruments are used. New diagnostic means are also developed, such as the one reported on in the prior section, and existing tests and instruments are continuously improved. A specific challenge for memory clinics, however, is that many 
Plejert, C., Jones, D. \& Peel, E. (2017). Pathways within dementia diagnosis: Conversation Analytical perspectives. In L-C. Hydén \& E. Antelius (Eds.) Life with Dementia: Relations, Responses and Agency in Everyday Life. London: Palgrave Macmillan. (C)

instruments, for example tests of cognitive functioning, are not adapted to people of all ethnicities, nor translated or validated in all languages needed, and rarely take limited literacy skills into account (e.g. Nielsen, 2011; Plejert et al., 2015). In addition, if a patient speaks a foreign language, it is routine in most memory clinics to appoint an interpreter to mediate the interaction between clinician and patient during the assessment. Although the field of research on interpreting in medical settings is vast (e.g. Angelelli, 2004; Karliner et al., 2007; Kaufert \& Putsch, 1997), there are very few studies on interpreting in settings involving people with dementia (but cf. Van De Mieroop et al., 2012), and even fewer on the specific environment of memory clinic encounters. However, in two case-studies of interpretermediated dementia evaluations, Plejert et al. (2015), and Majlesi and Plejert (2016) investigated the interplay between interlocutors in this particular setting. Whereas the first study (Plejert et al., 2015) highlighted particular challenges associated with the lack of cultural and linguistic adaptation of a test of cognitive functioning to the patient, and how this lack of adaptation played out in interaction, the second one (Majlesi \& Plejert, 2016) investigated how the clinician and interpreter used various embodied resources (e.g. pointing towards a watch, drawing a circle in the air as an iconic gesture for the face of a clock etc.) in collaborative ways that in various manners potentially affected the patient's understanding and performance of the task at hand. This section primarily discusses some key findings from the initial case-study (Plejert et al., 2015), but also highlights some further aspects of the data that formed the basis for that study. The data comprised video-recordings and ethnographic interviews collected in a Swedish memory clinic in 2013.

The participants in Plejert et al. (2015) are an experienced occupational therapist, a professional interpreter, and a patient, who is an 80 year-old Iraqi Kurdish woman. Her mother tongue is a variety of Kurdish called Feyli, but she also appears to know some Arabic and Turkish. She has limited reading and writing skills, and only knows how to count to a small extent. The interpreter is asked to translate between Swedish and Kurdish. The analysis primarily focuses on the administration of a test of cognitive functioning, the Montreal Cognitive Assessment (MoCA, Nasreddine, 2003). The MoCA is a screening instrument that assesses short-term memory (immediate and delayed recall of five nouns); visuospatial abilities (clock-drawing and three-dimensional cube-copy); executive functions (adapted Trail Making B, phonemic fluency and verbal abstraction); attention, concentration, and working memory (target detection, serial subtraction, digits forward and backwards); language (confrontation naming, repetition of two syntactically complex sentences, and phonetic fluency); and orientation in time and place. The test is available in many languages, however not Kurdish. The clinical staff in the memory clinic claimed that they experienced that the test most of the time worked reasonably well when mediated by an interpreter. In addition, and importantly, they were very cautious about basing their diagnosis on a combination of results from the overall assessment - the test of cognitive functioning was but one part out of many, and in case of any uncertainties, they would exclude results. However, as clinicians stated themselves, it was often fairly problematic for them to make informed inferences from patients' performances when interaction was mediated by an interpreter. 
Plejert, C., Jones, D. \& Peel, E. (2017). Pathways within dementia diagnosis: Conversation Analytical perspectives. In L-C. Hydén \& E. Antelius (Eds.) Life with Dementia: Relations, Responses and Agency in Everyday Life. London: Palgrave Macmillan. (C)

They felt, at times, uncertain about whether what they, or what the patient had said, had been rendered appropriately. As one physician said in an interview "we get what the patients are saying, but the nuances are missing”.

The skills and proficiency of the interpreter are naturally crucial for the process of assessment. Despite the fact that very many interpreters are highly skilled, there is, in Sweden and elsewhere, a lack of certified interpreters with a medical specialization in all languages (and varieties) required. And even with an advanced knowledge of medical interpreting, some tasks may be particularly demanding, such as interpreting that involves people with cognitive decline, such as dementia, or psychiatric conditions (cf. Bot 2003, 2005, on interpreting in mental health), where patients' contributions and responses may deviate from what is ordinarily expected. If the interpreter is not specifically trained to handle such contributions, it may be the case that the interpreter tries to make sense of what has been said, or selects to render what he/she perceives as "medically relevant" (Bolden, 2000).

It should be pointed out that very many interpreter-mediated dementia evaluations work very well. The following excerpt, however, demonstrates an example of the kind of challenges that all participants (not only the patient) are faced with, due to the fact that a test at hand is linguistically and culturally biased. Excerpts $4 \mathrm{a}-\mathrm{d}$ below come from the language part of the MoCA, and the patient is to repeat a syntactically complex sentence. In Swedish the sentence reads: "Jag vet att det är Johan som ska få hjälp idag", and in English: "I know that it is Johan who will get help today". The complexity lies in the original sentence comprising two subordinate clauses; a that clause (that it is Johan), and a relative clause (who will get help today). However, in Kurdish, relative clauses are not as common, and not always used in the same ways as in Germanic languages such as Swedish and English, and this potentially affects the interpreter's rendition (among other things). Right before Excerpt 4a starts, the occupational therapist (OT) has given the formal instructions, and these instructions have been rendered to the patient (PAT) in Kurdish by the interpreter (INT). The OT then produces the sentence to be repeated (line 01). A translation into idiomatic English is found in italics beneath each line of original language (Swedish, or Kurdish).

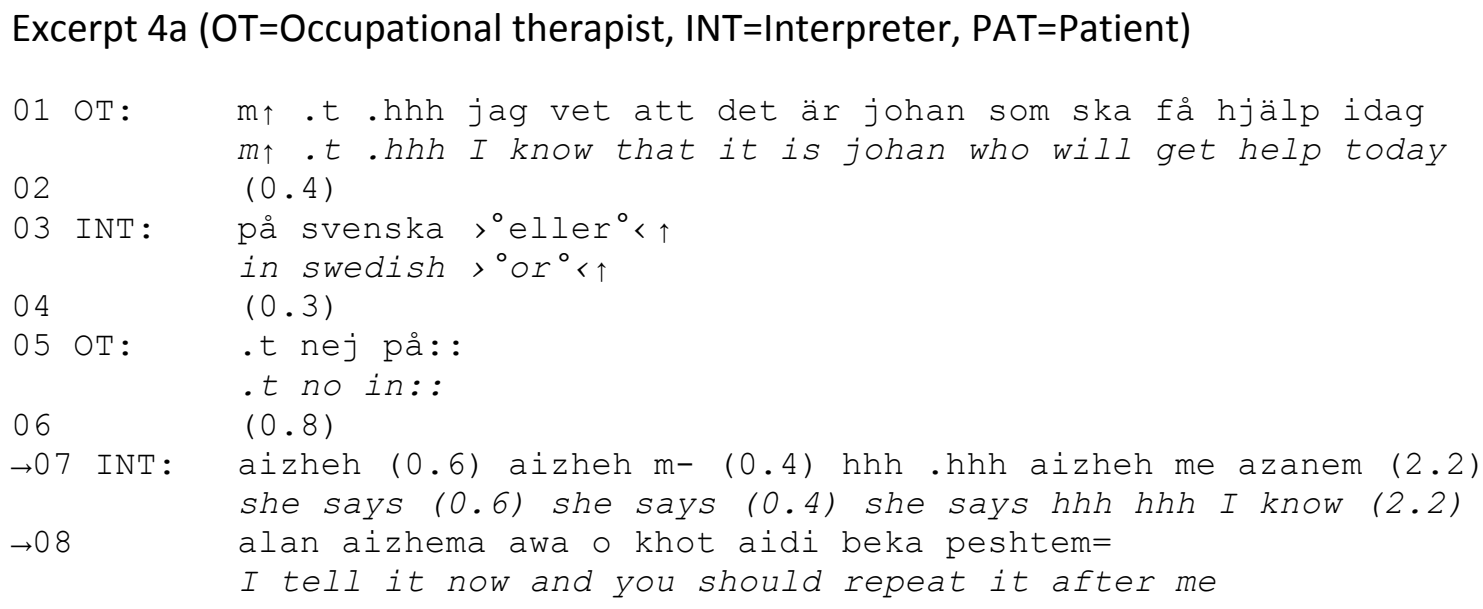


Plejert, C., Jones, D. \& Peel, E. (2017). Pathways within dementia diagnosis: Conversation Analytical perspectives. In L-C. Hydén \& E. Antelius (Eds.) Life with Dementia: Relations, Responses and Agency in Everyday Life. London: Palgrave Macmillan. (C)

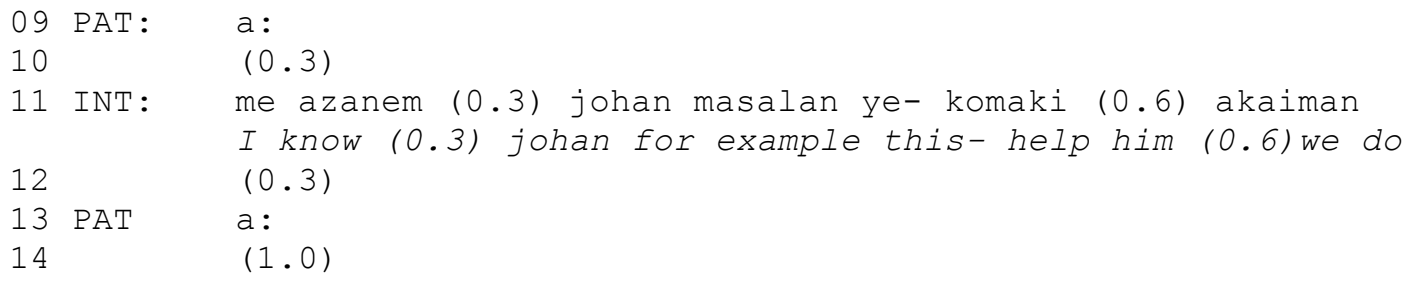

As can be observed, the interpreter is a bit confused whether the task is to be performed in Swedish or Kurdish (line 03), which may be due to the fact that the test material is in Swedish, so he must not only interpret, but also translate text at this point. Also, it is noticeable that after the nature of the task has been clarified, he once more repeats to the patient what the task is all about (lines 07-08), which is acknowledged by the patient (line 09). The interpreter's first version of the sentence to be repeated is then produced (line 11) "I know (0.3) Johan for example this-help him (0.6) we do". The fact that the word order is different in Kurdish is to be expected, since the two languages differ significantly in this respect (Kurdish having a less regulated word order), but already at this point, the complexity of the sentence has been somewhat reduced. The interpreter has also omitted the temporal term "today", which he notices, and then subsequently he provides a new sample sentence to be repeated. This is illustrated in Excerpt 4b:

\section{Excerpt $4 b$}

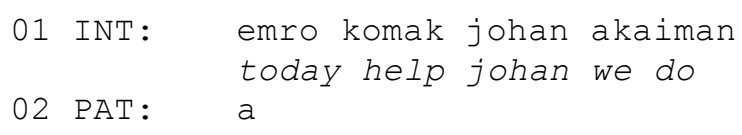

It can be noticed that in this second version (line 01 ), the syntactic complexity is entirely missing with no subordinate clauses. It should be added that the patient throughout this task has problems understanding what she is supposed to do. She continuously provides acknowledgment tokens as if she understands (e.g. line 02, Excerpt 4b, and line 13, Excerpt 4a), but does not repeat the test sentence until the interpreter finds out a way by asking the patient what he had just said (Excerpt 4c, line 03):

\section{Excerpt 4c}

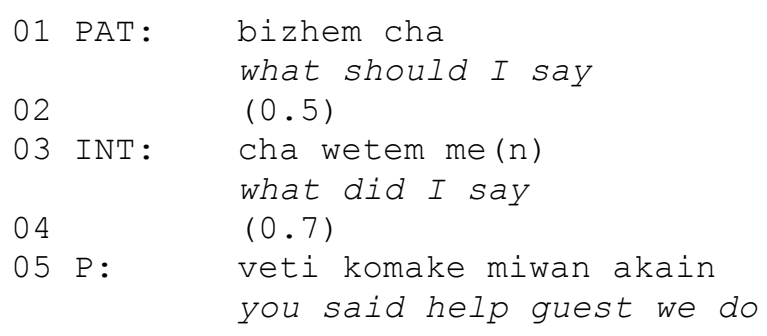

The patient eventually repeats the sentence (line 05 ). Her version is similar to the interpreter's (Excerpt 4b, line 01), apart from leaving out the temporal "today" and exchanging the name Johan to the Kurdish word "miwan", meaning "guest". It should be 
Plejert, C., Jones, D. \& Peel, E. (2017). Pathways within dementia diagnosis: Conversation Analytical perspectives. In L-C. Hydén \& E. Antelius (Eds.) Life with Dementia: Relations, Responses and Agency in Everyday Life. London: Palgrave Macmillan. (C)

noticed that prosodically, 'Johan' (the way the interpreter pronounces it) and 'miwan' share the same stress-pattern. In addition, the pronunciation of the second syllables of both words is the same. The fact that, throughout the interaction, the patient displays hearing difficulties may also have had an impact on what might be considered a rather qualified "guess" of a word that fits the overall meaning of the sentence.

The cultural bias of the task is also clearly visible here, since the name Johan does not exist in Kurdish. This fact might be an alternative explanation for the patient's attempt to find another word that fits the context. This leads to further repair-work between patient and interpreter, where the patient attempts to provide a name that exists in Kurdish, namely "Borhan":

\section{Excerpt $4 d$}

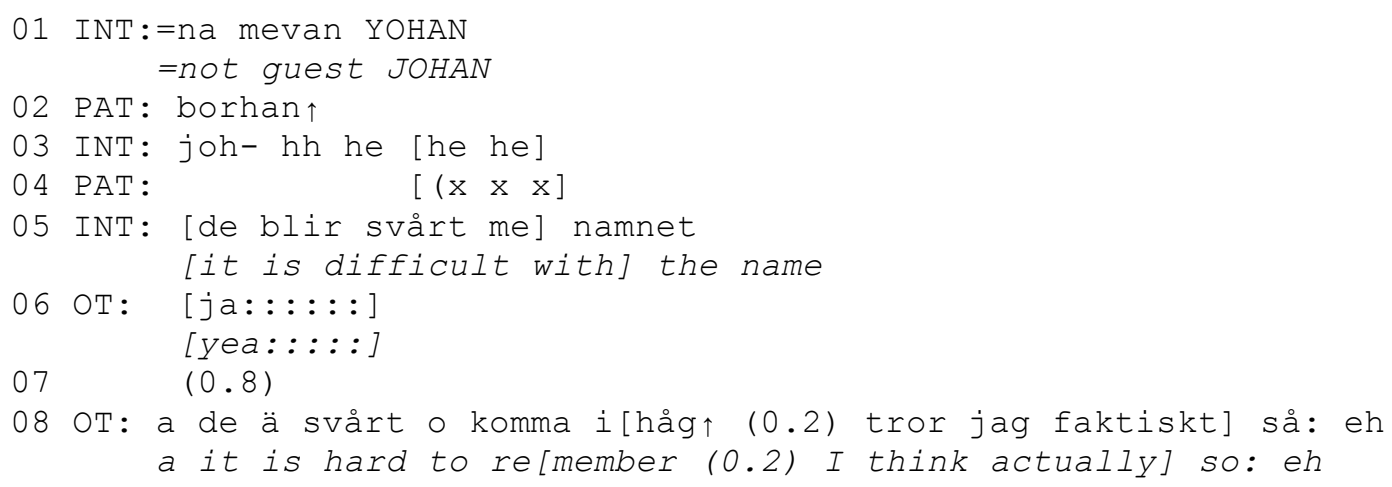

The interpreter performs cultural brokerage (e.g. Kaufert \& Koolage, 1984) and explains to the occupational therapist that the name Johan is difficult (line 05). However, at this point, and probably because the occupational therapist has not received sufficient renditions of the repair-work demonstrated in Excerpts 4a-c, she appears to interpret the patient's difficulties to perform the task as a memory difficulty (line 08 ).

\section{Diagnostic disclosure}

An important aspect of diagnostic pathways is the delivery of the diagnosis. As we discussed in the introduction, people may orient quite differently towards receiving a dementia diagnosis. Some are perhaps relieved and really want to know their diagnosis, whereas others might experience the diagnosis as stigmatizing or inappropriate. Some research suggests, however, that "catastrophic" reactions to a dementia diagnosis are fairly uncommon (e.g. Lecouturier et al., 2008). Historically, it was thought that people with dementia need not be well informed about the diagnosis or the disease. However, this attitude has now changed, and it is believed that a person with potential dementia "has the right to know" (Bamford et al., 2004; Hellström \& Torres, 2013, p. 158). Nonetheless, it is still the case that clinicians might tend to avoid explicitly using terminology such as "dementia" or "Alzheimer's Disease" with the patient themselves (Karneli-Miller et al., 2007; Peel, 2015). This is perhaps also reflected in the choice of the label "memory clinic" for specialist services, rather than more explicit terms such as "dementia service". Although, in the UK, for example, there has 
Plejert, C., Jones, D. \& Peel, E. (2017). Pathways within dementia diagnosis: Conversation Analytical perspectives. In L-C. Hydén \& E. Antelius (Eds.) Life with Dementia: Relations, Responses and Agency in Everyday Life. London: Palgrave Macmillan. (C)

been a move in some memory services to label them as more explicitly a dementia-related service, for instance the Worcestershire Early Intervention Dementia Service (La Fontaine et al., nd). In part, the use of the term dementia in the title of the service acts as a counter to the (potentially) obfuscatory and 'veiled' language often encountered in dementia health service provision (Karnieli-Miller et al., 2007). This degree of clarity about diagnostic service delivery is not, however, normative, and a fundamental issue is how is diagnostic disclosure communicated by clinicians, how it is it received by patients and relatives, and how can diagnosis 'best' be delivered.

The vast majority of research on dementia diagnostic disclosure is based on surveys, or in some cases interviews, primarily with clinicians (physicians, psychiatrists, neurologists etc.) or relatives of people with dementia, and in a few cases also turning to people with dementia (e.g. Clare, 2003) or people waiting to see a physician (Erde et al., 1998). A systematic review on dementia disclosure by Bamford et al. (2004) presented a highly intricate and complex picture of this field of research, outlining seven major themes: 1) should people with dementia be told their diagnosis?; 2) aspects of the process of disclosing and receiving a diagnosis of dementia; 3 ) studies of reported practice; 4) reasons for and against disclosure; 5) Factors influencing disclosure; 6) Impacts of disclosure; and 7) carers' experience of disclosure. From their review, Bamford et al. (2004, pp. 165-166) concluded that "for many practitioners, patients and carers, disclosing a diagnosis of dementia is neither inevitable nor straightforward" and they suggested that "qualitative research is needed to increase our understanding of the process of disclosure and enable people with dementia and their carers to obtain as much, or as little, information about their diagnosis as they want" (Ibid., p.167). While the question of whether people with a probable dementia should be told their diagnosis has largely receded from the academic literature and current western health care practice, there is great nuance and variability in how the diagnostic process proceeds, bearing in mind variations in individuals' cognitive and communicative abilities, degree of insight, and (potential) co-morbidities. Recent research using focus groups and interviews with current medical students, for example, highlights that deception remains a live issue in the context of dementia health care and that issues of capacity, perceived vulnerability and family dynamics all complicate clinical judgments in the dementia care context (Tullo et al., 2015); and this likely impacts on clinical communication.

A recent study that provides exactly the kind of empirical evidence called for by Bamford et al. (2004) is a CA-study by Peel (2015) on diagnostic communication in a memory clinic. As mentioned, CA methodology facilitates analyses of participants' moment-by-moment interactional practices and orientations. In interactions involving clinicians and patients with dementia and their relatives, it is therefore possible to closely study specific conversational actions, and how such actions are immediately responded and reacted to, for instance, the comparison between responses to questions by people with FMDs and people with dementia that we discussed above. Concerning questions such as, for example, whether a person with dementia should or should not be told their diagnosis, or, if clinicians avoid terms such as 
Plejert, C., Jones, D. \& Peel, E. (2017). Pathways within dementia diagnosis: Conversation Analytical perspectives. In L-C. Hydén \& E. Antelius (Eds.) Life with Dementia: Relations, Responses and Agency in Everyday Life. London: Palgrave Macmillan. (C)

"dementia" and "Alzheimer's disease", by means of CA it is possible to observe how these features are played out during real-time interaction. This is an important complement to results from survey and interview studies (particularly also since self-reports might not always reflect people's actual practices). The literature on clinician's self-reports of their diagnostic disclosure practices, for example, demonstrates that they are able to articulate their practices, such as putting a 'positive spin' on the diagnosis (Kissel \& Carpenter, 2007, p.277), but this does not offer a lens through which to scrutinize the process itself. Nor does this type of research illuminate how their professed approach to diagnostic delivery is received by patients and relatives.

Let us now turn to excerpts from Peel (2015) that demonstrate the actual process of diagnostic disclosure in a UK memory clinic. The data-set as a whole comprised videorecordings of 29 patients and accompanying persons attending a rural memory service, all of whom were white and native English speakers. Overall there were six interactions during which the old age psychiatrist was evaluating the patient's condition (Heritage \& Maynard, 2006). In Excerpts 5a-b below it is the patient's daughter (DAU) who seeks clarification about her mother's (PAT) brain scan.

Excerpt 5a (DR: Doctor, DAU: Daughter) (taken from Peel, 2015, p. 1125)

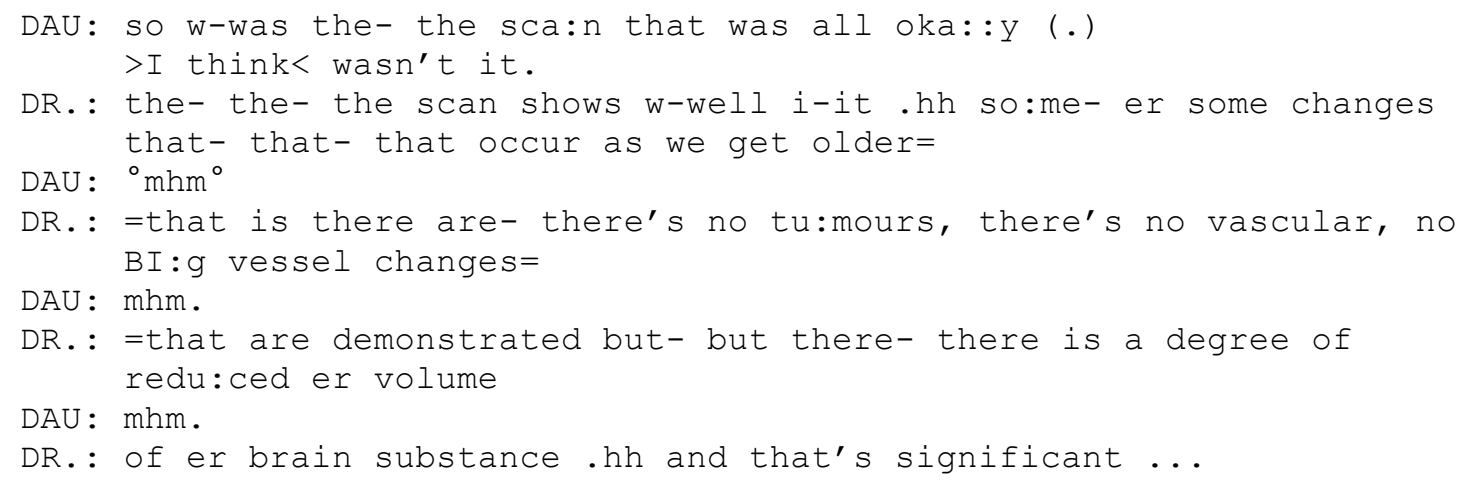

Here the daughter, starts to ask a question (so was the-) which she then self-repairs to a declarative question about the outcome of her mother's brain scan. When the doctor does not start his turn at 'okay' she downgrades her epistemic authority (Heritage \& Raymond, 2005) in deference to the doctor ('I think'). Through her reformulated question the daughter is seeking clarification on the outcome from the scan in a way that positions a problem as a dispreferred next turn ('all okay'), but also places the clinician in position of high epistemic authority. The diagnosis conveyed in lines 03-04, 06-07, 09-10 and 12 starts with 'well' which projects that the answer is not going to be straightforward (Schegloff \& Lerner, 2009) and initially normalises the scan results ('some changes that occur as we get older') and reports on what the scan did not find, before mentioning the 'significance' of reduced brain volume which is typical in Alzheimer's disease. We turn now to the close of this interaction.

Excerpt 5b (DR=Doctor, DAU: Daughter, PAT=Patient) 
Plejert, C., Jones, D. \& Peel, E. (2017). Pathways within dementia diagnosis: Conversation Analytical perspectives. In L-C. Hydén \& E. Antelius (Eds.) Life with Dementia: Relations, Responses and Agency in Everyday Life. London: Palgrave Macmillan. (C)

01 DAU: yep.

02 DR.: okay.

03 DAU: mhmm.

04 PAT: oh well $t^{\prime}$ hh thank you very much for your ti::me.

We can see in Excerpts 5a-b that, contrary to Karnieli-Miller et al.'s (2012) observation that by the end of the diagnostic encounter the person with dementia is being ignored, something more complex occurring. The doctor's shift in focus to the patient's daughter, rather than the patient herself, has been prompted by the daughter and the patient herself draws the exchange to a close. At this point the video shows the patient holding her head before moving her hand away as she says 'oh well' in line 04 , suggesting both a tacit acknowledgement of the information and (perhaps) an acceptance of its inevitability. But 'thank you very much for your time' arguably steps away from a more explicit articulation of receipt of this information (a thanking for information or expertise) through this more formulaic closing. These examples which show perhaps a normative degree of patient acknowledgement of diagnostic information, delivered with sensitivity to the concerns of the caring dyad rather than simply the patient as an individual. By contrast, the final example is taken from a consultation where the term 'Alzheimer's' is explicitly and unambiguously used by the clinician.

Excerpt 6: (DR=Doctor, PAT=Patient) (taken from Peel, 2015, p. 1127)

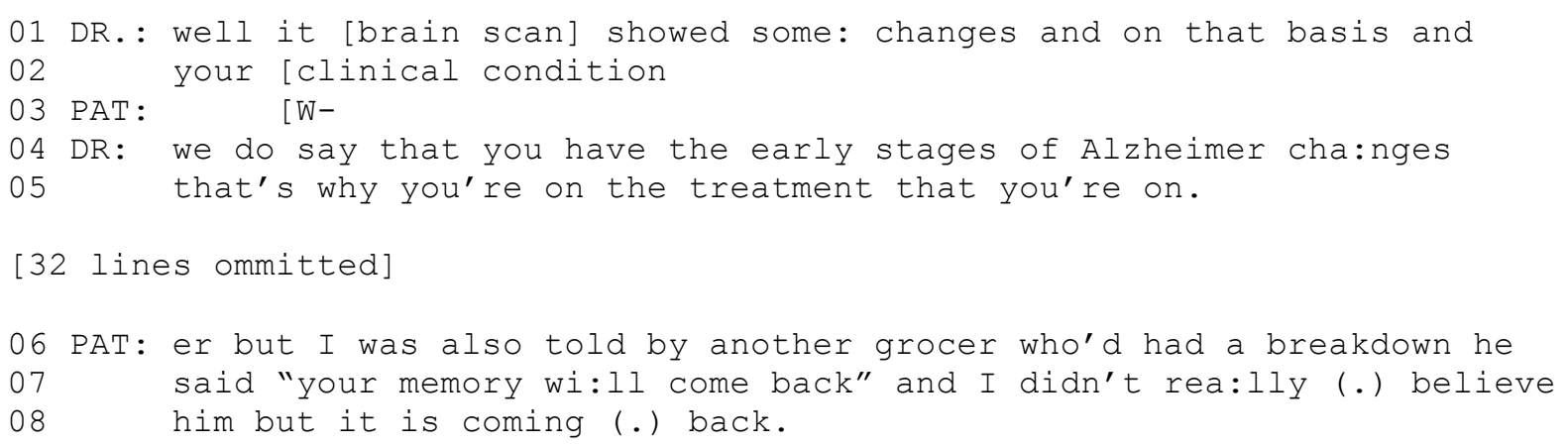

What this excerpt clearly highlights is that there is - at least for this patient at this time - no necessary correspondence between the explicit use of the term Alzheimer's and a recognition and understanding that a dementia is what he is experiencing. Therefore CA-informed analysis can bring into question the current assumption that the use of explicit terminology (e.g., Alzheimer's) is inherently important to the appropriate communication of diagnosis.

\section{Concluding discussion}

The aim of this chapter was to foreground different components of the dementia diagnosis pathway By way of discussion, we will explore more precisely the clinical and practical implications generated by the studies reported on above.

Beginning with the study by Jones et al. (2016), we showed how results from detailed analyses of interlocutors' interactional contributions may assist in diagnosing memory 
Plejert, C., Jones, D. \& Peel, E. (2017). Pathways within dementia diagnosis: Conversation

Analytical perspectives. In L-C. Hydén \& E. Antelius (Eds.) Life with Dementia: Relations, Responses and Agency in Everyday Life. London: Palgrave Macmillan. (C)

problems, and differentiating dementia from non-organic FMD. These findings, and the resulting profiles, could underpin an interactional toolkit to aid practitioners in designing their consultation to maximize diagnostic potential, and provides concrete grounds to support clinicians" intuitive formations of a "working diagnosis" during the first couple of minutes of an initial consultation. It should be pointed out, however, that the study reports initial observations that need to be developed further to become an additional diagnostic method in memory clinics. To date these candidate diagnostic interactional features have been developed into a working diagnostic scoring tool and blind tested by two linguistic coders against ten patients (five with confirmed FMD diagnosis and five with dementia) to check inter-rater reliability and the diagnostic accuracy of the distinctive conversational profiles (Elsey et al., 2015). The results appear promising, particularly in terms of enabling a more speedy diagnosis, and for potentially sparing patients extensive and burdensome neuropsychological testing. Additionally, the toolkit could be used in other services within the diagnostic pathway including GP's in primary care who are increasingly required to 'screen' for dementia. It may help to ensure patients are being referred to appropriate secondary services (e.g. counseling services for patients with functional memory complaints resulting from anxiety or depression) and reduce the burden on memory clinic services. Using conversation as a method of assessment throughout the assessment pathway could therefore be of significant diagnostic value.

When it comes to the study of the interpreter-mediated dementia assessments, it demonstrates the very many interactional details that may influence not just the actual administration of different parts of cognitive evaluation, but also how, for example, patients' performances on different parts of a test are to be assessed. In Excerpts 4a-d, the interpreter and the patient got involved in a series of repair-sequences concerning the patient's lack of understanding of the task, her potential hearing difficulty, and the fact that the name to be repeated was (culturally) unfamiliar to her. In addition, the interpreter appeared strongly oriented towards making the patient carry out the task in the way asked for by the occupational therapist. This potentially contributed to his not consistently rendering what was being said during the repair. Having access to the transcript, however, analysis revealed that several interactional actions were of potentially great relevance from a diagnostic point of view, details unavailable for the clinician. Also, despite the fact that the interpreter did attempt to explain that the culturally unfamiliar name was probably contributing to the patient's difficulties, the lack of access to what had been going on during repair, eventually influenced the occupational therapist's interpretation of the patient's performance of the task. These findings suggest that optimally, interpreter-mediated encounters should be recorded, transcribed and translated, and transcriptions subsequently screened by the clinicians involved in the evaluation. However, such a process would be way too costly and time-consuming, and also ethically problematic, since all patients would perhaps not give their consent to being audio- or video recorded. The findings also support current developments of tests that are more adapted to patients with limited reading and writing skills, and less culture dependent, for example the Rowland Universal Assessment Scale, RUDAS (see Naqvi et al., 2015). To use such tests probably 
Plejert, C., Jones, D. \& Peel, E. (2017). Pathways within dementia diagnosis: Conversation

Analytical perspectives. In L-C. Hydén \& E. Antelius (Eds.) Life with Dementia: Relations, Responses and Agency in Everyday Life. London: Palgrave Macmillan. (C)

also facilitates the interpreters' task. This, however, remains to be investigated further, preferably using CA. Finally, our observations also signal that further interpreter training is needed, particularly concerning formal medical tasks, such as neuropsychological tests. In relation to the current steep increase in Sweden, and world-wide, in immigrant populations, there are discussions (in Sweden) at the national level concerning developing special 'shortcuts' in interpreter training. This is worrying, considering the challenges already facing interpreters in terms of often not having enough training for mediating talk during quite specific clinical tasks, or, encounters involving people with potential cognitive impairments. If anything, more training is needed, rather than less, to secure assessment accuracy.

When it comes to dementia disclosure, we demonstrated in Excerpts 5a-b how the diagnosis may be communicated in rather a "veiled" way (Bergmann, 1992), as the seriousness of the diagnosis is communicated through the words 'significant' and 'changes' rather than through the direct use of terms like Alzheimer's disease. Overall, this example would suggest that while explicit diagnostic information is not delivered (i.e., probable Alzheimer's disease) the receipt of the information is in step with diagnostic delivery as it occurs in other medical settings (i.e., minimal) and conforms to the intersubjective norms of minimizing catastrophic reactions in medical communication (Maynard \& Frankel, 2006). Rather than dementia diagnosis delivery necessarily being a "special case", in keeping with the receipt of diagnostic information in other medical contexts (e.g., Heath 1992) this information is not received as news, but rather is minimally receipted by the daughter and met with silence from the patient. Nevertheless, these interactions highlight not only the considerable complexity at work within this context but also knotty interpersonal and family dynamics, which are being managed on an unfolding basis (Peel, 2015; Riggs \& Peel, 2016). By contrast, Excerpt 6 demonstrated that the use of explicit diagnostic terms does not necessarily improve patients' understanding or awareness of their condition. Therefore CA analyses may enable us to trouble the notion in the existing literature (e.g., Karnieli-Miller et al., 2007) that health professionals' not typically using the words Alzheimer's, or dementia in interactions with patients as being fundamentally problematic and obfuscatory, and further a more nuanced understanding of the interactional processes involved in communicating diagnosis. How these interactional processes map onto different 'types' of patients and caring dyads or triads, in terms of being mediated by culture/ethnicity, gender, social class, age, and level of (dis)ability is yet to be charted. Yet, as the above examples across the three key aspects of dementia diagnostic practice (initial assessment, cognitive testing, and diagnostic delivery) attest, CA offers a rich analytic seam through which to mine, and ultimately inform, diagnostic pathways and practices in dementia care.

As our discussion of these three aspects within the dementia diagnosis pathway has highlighted, a close focus on how, and in what ways, interactions unfold can illuminate a range of components in dementia care. By closely exploring dementia care as it is actually enacted (rather than relying on second-hand reports) there is the potential to understand how the diagnostic experience can be improved for health professionals, family carers, and most 
Plejert, C., Jones, D. \& Peel, E. (2017). Pathways within dementia diagnosis: Conversation Analytical perspectives. In L-C. Hydén \& E. Antelius (Eds.) Life with Dementia: Relations, Responses and Agency in Everyday Life. London: Palgrave Macmillan. (C)

importantly for people living with dementia themselves. Understanding how diagnostic interactions work in practice can potentially assist in improving practice. Ultimately, improved practice impacts on better patient experience, which may contribute to better adjustment to living with dementia in the long term.

\section{Transcription conventions (adapted from Jefferson, 2004)}

$=\quad$ Links talk produced in close temporal proximity (latched talk)

$\quad>\quad$ Talk between symbols is rushed or compressed

$\circ \quad$ Encloses talk which is produced quietly

underline Underlining marks emphasis of some kind

CAPS Words or parts of words spoken loudly marked in capital letters

s::::: $\quad$ Sustained or stretched sound; the more colons, the longer the sound

. ?, $\quad$ Stop indicates falling intonation; a question mark indicates rising intonation over a word; a comma indicates a slight rising intonation a the end of word

.hhh Inbreath, the number of 'h's representing the length of the inbreath

hhh. Outbreath, the number of 'h's representing the length of the outbreath

[] Encloses talk in overlap i.e. when more than one speaker is speaking

(word) Parentheses indicate transcriber doubt

(this/that) Alternative hearings

((description)) Description of what can be heard, rather than transcription e.g.

((shuffling papers))

cu- Cut-off word or sound

(0.6) Silence in seconds

(.) Silence of less than two tenths of a second

$\wedge$ or $\uparrow \quad$ Indicates marked pitch rise

$\vee$ or $\downarrow \quad$ Indicates marked fall in pitch

(hhenhh) Indicates laughter while speaking (aspiration)

\section{Acknowledgments}

The research on which this chapter is based was funded by the Bank of Sweden Tercentenary Foundation, RJ (grant number M10-0187:1), the National Institute for Health Research (NIHR) under its Research for Patient Benefit (RfPB) Programme [grant number PB-PG0211-24079], and a British Academy Mid-Career Fellowship [grant number MC110142].

\section{References}

Adelman, A.M. \& Daly, M. P. (2005). Initial evaluation of the patient with suspected dementia. American Family Physician, 1, 71 (9):1745-1750.

Angelelli, C. V. (2004). Medical Interpreting and Cross-Cultural Communication. Cambridge: Cambridge University Press.

Antaki, C. (2011). Applied Conversation Analysis. Basingstoke: Palgrave-Macmillan. 
Plejert, C., Jones, D. \& Peel, E. (2017). Pathways within dementia diagnosis: Conversation

Analytical perspectives. In L-C. Hydén \& E. Antelius (Eds.) Life with Dementia: Relations, Responses and Agency in Everyday Life. London: Palgrave Macmillan. (C)

Bamford, C., Lamont, S., Eccles, M., Robinson, L., May, C. \& Bond, J. (2004). Disclosing a diagnosis of dementia: a systematic review. International Journal of Geriatric Psychiatry, 19: 151-169.

Bergmann, J. R. (1992). Veiled morality: notes on discretion in psychiatry. In P. Drew \& J. Heritage (Eds.). Talk at Work (pp. 137-162). Cambridge: Cambridge University Press. Blackburn, D.J., Wakefield, S., Shanks, M.F., Harkness, K., Reuber, M., \& Venneri, A. (2014). Memory difficulties are not always a sign of incipient dementia: A review of the possible causes of loss of memory efficiency. British Medical Bulletin, 112: 71-81.

Bolden, B. G. (2000). Towards understanding practices of medical interpreting: Interpreters' involvement in history taking. Discourse Studies, 2 (4): 387-419.

Bot, H. (2003). The myth of the uninvolved interpreter in mental health and the development of a three-person psychology. In. L- Brunette, G. Bastin, I.Hemlin \& H. Clarke (Eds.). (pp.27-35). The Critical Link3, Amsterdam/Philadelphia: John Benjamins.

Bot, H. (2005). Dialogue Interpreting in Mental Health, Amsterdam/ New York: Rodopi Publishers.

Brooker, D., La Fontaine, J., Evans, S., Bray, J. \& Saad, K. (2014). Public health guidance to facilitate timely diagnosis of dementia: Alzheimer's Cooperative Valuation in Europe recommendations. International Journal of Geriatric Psychiatry, 29: 682-693.

Chatwin, J. (2014). Conversation analysis as a method for investigating interaction in care home environments. Dementia, 13 (6): 737-746.

Clare, L. (2003). Managing threats to self: awareness in early stage Alzheimer's disease. Social Science \& Medicine, 57: 1017-1029.

Dilworth-Anderson, P., \& Gibson, B. (2002). The cultural influence of values, norms, meanings, and perceptions in understanding dementia in ethnic minorities. Alzheimer Disease and Associated Disorders, 16: 56-63.

Dilworth-Anderson, P., Williams, I. C., \& Gibson, B. F. (2002). Issues of race, ethnicity, and culture in caregiving research: A 20-year review (1980-2000). The Gerontologist, 42 (2): 237-272.

Drew, P. (2005). Conversation analysis. In K. L. Fitch, \& R. E.Sanders (Eds.). Handbook of Language and Social Interaction (pp. 71-102). London: Lawrence Erlbaum Associates Publishers.

Elsey, C., Drew, P., Jones, D., Blackburn, D., Wakefield, S., Harkness, K., Venneri, A. \& Reuber, M. (2015). Towards diagnostic conversational profiles of patients presenting with dementia or functional memory disorders to memory clinics. Patient Education and Counseling, 98 (9):1071-7.

Erde, E. L., Nadal, E. C. \& Scholl, T. O. (1988). On truth telling and the diagnosis of Alzheimer's disease. Journal of Family Practice, 26:401-406.

Erol, R., Brooker, D. \& Peel, E. (2015). Women and Dementia: A global research review. London: Alzheimer's Disease International. Available at: http://www.alz.co.uk/women-and-dementia 
Plejert, C., Jones, D. \& Peel, E. (2017). Pathways within dementia diagnosis: Conversation

Analytical perspectives. In L-C. Hydén \& E. Antelius (Eds.) Life with Dementia: Relations, Responses and Agency in Everyday Life. London: Palgrave Macmillan. (C)

Erol, R., Brooker, D. \& Peel, E. (2016). The impact of dementia on women internationally: An integrative review. Health Care for Women International. doi:10.1080/07399332.2016.1219357 Advance access.

Goodwin. C. (2003). Conversation and Brain Damage. Oxford: Oxford University Press.

Heath, C. (1992). The delivery and reception of diagnosis and assessment in the general practice consultation. In P. Drew \& J. Heritage (Eds.), Talk at Work (pp. 235-267). Cambridge: Cambridge University Press.

Hellström, I. \& Torres, S. (2013). A wish to know but not always tell - couples living with dementia talk about disclosure preferences. Aging \& Mental Health, 17 (2): 157-167. DOI:10.1080/13607863.2012.742491.

Heritage, J. \& Raymond, G. (2005). The terms of agreement: Indexing epistemic authority and subordination in talk-in-interaction. Social Psychology Quarterly, 68 (1): 15-38.

Heritage, J. \& Maynard, D. W. (2006). Introduction: Analyzing interaction between doctors and patients in primary care encounters. In J. Heritage \& D. W. Maynard (Eds.). Communication in Medical Care. (pp. 1-21) New York: Cambridge University Press.

Heritage, J., Robinson, J., Elliott, M., Beckett, M., Wilkes, M. (2007). Reducing patients' unmet concerns in primary care: the difference one word can make. Journal of General Internal Medicine, 22:1429-33.

Hydén, L-C., Samuelsson, C., Örulv, L. \& Plejert, C. (2012). Feedback in narrative interaction involving people with dementia. Journal of Interactional Research in Communication Disorders, 4 (2): 211-247.

Jefferson, G. (2004). Glossary of transcript symbols with an introduction. In G. H. Lerner (Ed.). Conversation analysis: Studies from the First Generation (pp. 13-23). Philadelphia, PA: John Benjamins.

Jones, D. (2013). A family living with Alzheimer's disease: The communicative challenges. Dementia: The International Journal of Social Research and Practice. Advance online publication. doi:10.1177/1471301213502213.

Jones, D., Drew, P., Elsey, C., Blackburn, D., Wakefield, S., Harkness, K. \& Reuber, M. (2016). Conversational assessment in memory clinic encounters: interactional profiling for differentiating dementia from functional memory disorders. Aging \& Mental Health, 20 (5): 500-509.

Karliner, L. S., Jacobs, E. A., Hm Chen, A. \& Mutha, S. (2007). Do professional interpreters improve clinical care for patients with limited English proficiency? A systematic review of the literature. Health Services Research, 42 (2): 727-748.

Karnieli-Miller O. Werner P. Aharon-Peretz J. Eidelman S. (2007). Dilemmas in the (un)veiling of the diagnosis of Alzheimer's disease: Walking an ethical and professional tight rope. Patient Education and Counseling, 67: 307-314.

Karnieli-Miller O. Werner P. Neufeld-Kroszynski G. Eidelman S. (2012). Are you talking to me?! An exploration of the triadic physician-patient-companion communication within memory clinics encounters. Patient Education and Counseling, 88 (3): 381-390. 
Plejert, C., Jones, D. \& Peel, E. (2017). Pathways within dementia diagnosis: Conversation

Analytical perspectives. In L-C. Hydén \& E. Antelius (Eds.) Life with Dementia: Relations, Responses and Agency in Everyday Life. London: Palgrave Macmillan. (C)

Kaufert, J. \& Putsch, R. (1997). Communication through interpreters in healthcare: Ethical dilemmas arising from differences in class, culture, language and power. The Journal of Clinical Ethics, 8 (81): 71-87.

Kaufert, J. M. \& Koolage, W.W. (1984). Role conflict among "culture brokers": The experience of native Canadian medical interpreters. Social Science and Medicine, 18: 283-286.

Kissel E. C. \& Carpenter B. D. (2007). It's all in the details: Physician variability in disclosing a dementia diagnosis. Aging \& Mental Health, 3:273-280.

LaFontaine, J., Brooker, D., Wallcraft, J. \& Vickers, H. (nd) Evaluation Report Early Intervention Dementia Service Worcestershire. University of Worcester: Association for Dementia Studies.

Lecouturier, J., Bamford, C., Hughes, J. C., Francis, J. J., Foy, R. \& Eccles, P. M. (2008). Appropriate disclosure of a diagnosis of dementia: identifying the key behaviours of 'best practice'. BMC Health Services Research, 8: 95. DOI: 10.1186/1472-6963-8-95.

Leibing, A. \& Cohen, L. (2006). Thinking about Dementia. Culture, Loss, and the Anthropology of Senility. New Jersey: Rutgers University Press.

Linell, P., Hofvendahl, J. \& Lindholm, C. (2003). Multi-unit questions in institutional interactions: Sequential organizations and communicative functions. Text, 23, 4: 539571.

Majlesi, A R. \& Plejert, C. (2016). Embodiment in tests of cognitive functioning: A study of an interpreter-mediated dementia evaluation. Dementia. DOI: $101177 / 1471301216635341$.

Maynard, D. W. \& Frankel, R. M. (2006). On diagnostic rationality: bad news, good news, and the symptom residue. In J. Heritage \& D. W. Maynard (Eds.). Communication in Medical Care. (pp. 248-278). New York: Cambridge University Press.

Nasreddine, Z. (2003-2014). The Montreal Cognitive Assessment MoCAC.

Naqvi, M.R; Halder, S., Tomlinson, G. \& Alibhai, S. (2015). Cognitive assessments in multicultural populations using the Rowland Universal Dementia Assessment Scale; A systematic review and meta-analysis. Canadian Medical Association Journal Early release, www.cmaj.ca, February 17, 2015. CMAJ 2015. DOI:10.1503 /cmaj.140802.

Nielsen, T. R. (2011). Evaluation of Dementia in Patients from Ethnic Minorities. A European Perspective. Doctoral Dissertation. Copenhagen University.

O'Connor, D.W., Pollitt, P.A. \& Treasure, F.P. (1991). The influence of education and social class on the diagnosis of dementia in a community population. Psychological Medicine 21: 219-224.

Peel, E. (2014). 'The living death of Alzheimer's' versus 'Take a walk to keep dementia at bay': Representations of dementia in print media and carer discourse. Sociology of Health and Illness 36 (6): 885-901.

Peel, E. (2015). Diagnostic communication in the memory clinic: A conversation analytic perspective. Aging \& Mental Health 19 (12): 1123-1130. 
Plejert, C., Jones, D. \& Peel, E. (2017). Pathways within dementia diagnosis: Conversation

Analytical perspectives. In L-C. Hydén \& E. Antelius (Eds.) Life with Dementia: Relations, Responses and Agency in Everyday Life. London: Palgrave Macmillan. (C)

Peel, E. \& Harding, R. (2014). "It's a huge maze, the system, it's a terrible maze": Dementia carers' constructions of navigating health and social care services. Dementia: The International Journal of Social Research and Practice, 13(5) 642-666.

Peel, E. \& McDaid, S. (2015). 'Over the Rainbow': Lesbian, gay, bisexual, trans people and dementia project. Summary Report. University of Worcester. Available at: http://dementiavoices.org.uk/wp-content/uploads/2015/03/Over-the-RainbowLGBTDementia-Report.pdf

Plejert, C., Antelius, E., Yazdanpanah, M. \& Nielsen, T. R. (2015). There's a letter called ef. On challenges and repair in interpreter-mediated tests of cognitive functioning in dementia evaluations: A case study. Journal of Cross-Cultural Gerontology, 30 (2): 163-187.

Plug, L., Sharrack, B. \& Reuber. M. (2011). Metaphors in the description of seizure experiences: common conceptualisations and differential diagnosis. Language and Cognition, 3-2: 209-233.

Reuber, M., Monzoni, C., Sharrack, B. \& Plug, L. (2009). Using Conversation Analysis to distinguish between epileptic and psychogenic non-epileptic seizures: a prospective blinded multi-rater study. Epilepsy and Behavior, 16:139-144.

Riggs, D.W. \& Peel, E. (2016). Critical Kinship Studies: An introduction to the field. London: Palgrave Macmillan.

Robson, C., Drew, P., Walker, T., Reuber, M. (2012). Catastrophising and normalising in patient's accounts of their seizure experiences. Seizure, 21:795-801.

Rosser, M.N., Fox, N.C., Mummery, C.J., Schott, J.M. \& Warren, J. D. (2010). The diagnosis of young-onset dementia. Lancet Neurology 9: 973-806.

Royal College of Psychiatrists (2013). English national memory clinic audit report. Retrieved from http://www.rcpsych.ac. uk/pdf/English\%20National\%20Memory\%20Clinics\%20 Audit\%20Report\%202013.pdf

Sacks, H., Schegloff, E. A. \& Jefferson, G. (1974). A simplest systematics for the organization of turn-taking for conversation, Language 50 (4): 696-735.

Samsi, K., Abley, C., Campbell, S., Keady, J., Manthorpe, J., Robinson, L., Watts. \& Bond, J. (2014). Negotiating a labyrinth: Experiences of assessment and diagnostic journey in cognitive impairment and dementia. International Journal of Geriatric Psychiatry 29: 58-67.

Schegloff, E. A. (1996). Confirming allusions: Toward an empirical account of action. American Journal of Sociology, 104 (1): 161-216.

Schegloff, E. A. (2007). Sequence Organization in Interaction. A Primer in Conversation Analysis. Cambridge : Cambridge University Press.

Schegloff, E. A., Jefferson, G. \& Sacks, H. (1977). The preference for self-correction in the organization of repair in conversation. Language, 53 (2): 361-382).

Schegloff, E. A. \& Lerner, G. H. (2009). Beginning to respond: Well-prefaced responses to wh-questions. Research on Language and Social Interaction, 42(2): 91-115. 
Plejert, C., Jones, D. \& Peel, E. (2017). Pathways within dementia diagnosis: Conversation Analytical perspectives. In L-C. Hydén \& E. Antelius (Eds.) Life with Dementia: Relations, Responses and Agency in Everyday Life. London: Palgrave Macmillan. (C)

Schrauf, R. W. \& Iris, M. (2011). Very long pathways to diagnosis among African Americans and Hispanics with memory and behavioral problems associated with dementia. Dementia, 11 (6): 743-763.

Schmidtke, K., Pohlmann, S., \& Metternich, B (2008). The syndrome of functional memory disorder: Definition, etiology, and natural course. American Journal of Geriatric Psychiatry, 16, 981-988.

Shadlen, M-F., Larson, E. B., Gibbons, L., McCormick, W. C. \& Teri, L. (1999). Alzheimer's disease symptom severity in Blacks and Whites. American Geriatrics Society 47 (4): 482-486.

Sidnell, J. (2010). Conversation Analysis: An Introduction. Sussex: Wiley-Blackwell.

Sidnell, J. \& Stivers, T. (2013). The Handbook of Conversation Analysis. Chichester: WileyBlackwell.

Stivers, T. (2007). Prescribing under Pressure: Parent-Physician Conversations and Antibiotics. Oxford: Oxford University Press.

Tullo, E. S., Lee, R. P., Robinson, L. \& Allan, L. (2015). Why is dementia different? Medical students' views about deceiving people with dementia. Aging and Mental Health, 19 (8): 731-738.

Van De Mieroop, D., Bevilacqua, G. and Van Hove, L. (2012). Negotiating discursive norms. Community interpreting in a Belgian rest home. Interpreting,14 (1): 23-54. 